\title{
Squamous Cell Carcinoma in a Dog (Canis familiaris) Treated by the Viscum album Therapy - Case Report
}

\author{
Ana Catarina Viana Valle ${ }^{1,2 *}$ and Aloisio Cunha de Carvalho ${ }^{1,3}$ \\ ${ }^{1}$ Doctor Izao Soares Institute, Integrative Medicine, Ribeirao Preto, SP, Brazil \\ ${ }^{2} \mathrm{PhD}$ in Genetics and Biotechnology, Catholic University of Brasilia, Brazil \\ ${ }^{3} \mathrm{PhD}$ in Ambiental Pathology, Paulista University, Brazil
}

${ }^{\star}$ Corresponding author: Dr. Ana Catarina Viana Valle, Doctor Izao Soares Institute, Integrative Medicine, Ribeirao Preto, SP, Brazil; Email: dranacatarina@gmail.com Received: June 04, 2021; Accepted: June 15, 2021; Published: June 17, 2021

\begin{abstract}
Squamous Cell Carcinoma is defined as a common malignant neoplasm in the clinical routine of small animals. The treatment of choice includes surgical removal, chemotherapy, radiotherapy, cryosurgery, most of which result in mutilation, deformation, side effects, metastases, and recurrences. Therapy failure is reported by several authors and depends on the disease stage and the patient's age and health condition. The need for developing new therapies for cancer treatment is well-known, aiming the prophylaxis, treatment, and, when possible, cure of the disease. Under this scope, the Viscum album therapy has been used for more than 100 years by human medicine with reported success in several types and stages of cancer. Therefore, this work describes the administration of ultra-diluted Viscum album in a dog diagnosed with oral SCC. The disease was successfully cured, which was later confirmed by histopathology analysis.
\end{abstract}

Keywords: Cancer, Viscum album, Dog, Therapy

\section{Introduction}

Squamous Cell Carcinoma (SCC) is defined as a relatively common malignant neoplasm, accounting for $5 \%$ of all skin tumors. It is considered the second most common oral cancer type (31\%) in dogs and cats [1], behind oral melanoma (44\%) and prior to oral fibrosarcoma (25\%) [2]. However, SCC can arise in various locations in the body $[1,3]$. In humans, it accounts for $20 \%$ of all skin cancers, resulting in 1 million cases in the United States each year. Most cutaneous SCCs are treated locally. However, there is a high incidence of recurrences, metastases, followed by death [3].

The SCC resulting from the oral mucosa epithelium is locally invasive, being considered a severe disease and directly dependent on the stage of the disease's evolution. It is considered a deforming pathology due to the rapid infiltration of neoplastic cells in the tissues, orofacial destruction, and possible metastases [4].

The SCC etiology is probably multifactorial. Several risk factors are involved, including the use of flea collars and history of industrialized feeding in cats $[1,5]$.

The treatment of choice for oral SCC is surgical, which is considered the best option. However, most of the time, patients are mutilated [5]. Early SCC diagnosis and treatment are essential since initial stage tumors are the most amenable to treatment and with the best prognosis [1]. Surgery, radiotherapy, chemotherapy, and their combinations are also possible options for this treatment. Nevertheless, they have already been tested and rarely presented a satisfactory response. An integrative treatment approach appears to be a better option for successful therapy in these cases [5]. In addition, one must consider animals in advanced stages of the disease and those in palliative care. These patients can benefit from such therapies with regard to improving their quality of life until their death, providing them dignity. In situations like those mentioned above, an approach within integrative therapies would probably offer a better chance of success due to the limitations of the treated patients [5].

Within this context, Valle and Carvalho [6] refer to administering the Viscum album therapy for prophylaxis, treatment and/or cure of cancer patients in its various stages, including animals in palliative care. Viscum album is an excellent treatment option for cancer patients as it has a bidirectional action stimulating the immune system of patients and selectively exerting cytotoxicity against tumor cells [7]. Besides that, it does not have side effects on the body. For this reason, the Viscum album therapy has been used for more than 100 years by human medicine with reported success in several types and stages of cancer [8]. This study aimed to report the case of a 16-year-old female dog with a diagnosis of oral SCC, treated using an ultra-diluted Viscum album.

\section{Case Report}

A 16-year-old female Maltese dog weighing $3.9 \mathrm{~kg}$, fed with commercial food, was seen at NaturalPet Clinic in Brasilia, DF, Brazil. The patient was diagnosed with a well-differentiated SCC (60\%; Figure 1) four weeks before the veterinary appointment at NaturalPet. The biopsy report carried out by the Histopato Laboratory (Brasilia, DF, Brazil) details that approximately $60 \%$ of the analyzed fragment was affected by neoplastic, hypercellular, homogeneous, infiltrated, not 


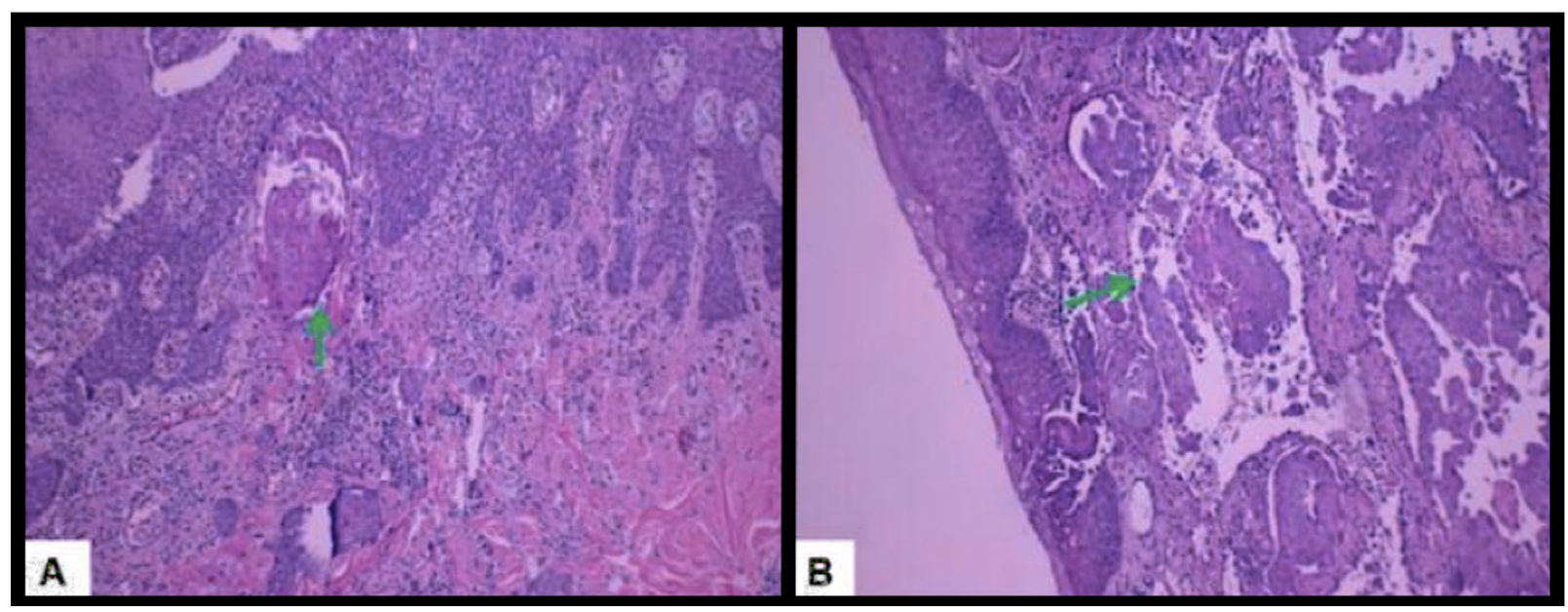

Figure 1: Photomicrograph A. Arrow: Keratin pearl. B. Arrow: Vascular involvement by neoplasm (Photo: Laboratory of Pathology - Histopato, Brasilia-Brazil).

well-demarcated, and non-encapsulated lesion. The neoplastic cells were arranged in cohesive blocks bordering concentric and cohesive keratin sheets (keratin pearl). The cells were polygonal with moderate and eosinophilic cytoplasm, rounded nucleus, dispersed chromatin, single and evident nucleolus. Vessels and margins were affected by neoplastic cells.

On physical examination, the patient showed aggressive behavior and, at the slightest attempt to touch, she already reacted in a very hostile way. Therefore, the examination was performed very carefully. The animal had normal-colored mucous membranes, CPT 2", cardiac and respiratory auscultation within the expected range for age and species, hydration conditions within the normal range, moderate sialorrhea accompanied by halitosis. An ulcerated lesion was observed, and the fur was stained with blood around it. The lesion was located below the upper lip, exacerbating gingival mass (Figure 2). The impossibility of manipulating the patient made the examination difficult, but the tutor helped with the procedure. A protocol was instituted using the Viscum album therapy, and it consisted of the first application of Viscum album D3 (1 X 10-3), intravenously, every seven days, for four weeks, associated with the administration of Viscum album D3, subcutaneously, one ampoule $(1.1 \mathrm{~mL})$, once a day, on alternate days, initially for 30 days. The subcutaneous applications were performed by the tutor. In addition, it was recommended to replace the commercial diet with a natural diet consisting of a low-carbohydrate recipe (15\%). Blood was also collected for laboratory tests of complete blood count and biochemical measurements of alanine aminotransferase (ALT), alkaline phosphatase (AP), urea, and creatinine.

\section{Results}

The results of the blood tests showed - Red blood cells: 7,200,000/ uL; Hemoglobin: 15.9 g/dL; Hematocrit: 45\%; MCV: 62.5 fL; MCHC: 35.33 g/dL; Leukocytes: 27.400/uL; Platelets: 308.000/uL; ALT - 58 U/L; AP: $61 \mathrm{U} / \mathrm{L}$; Creatinine: $0.61 \mathrm{mg} / \mathrm{dL}$; Urea: $32 \mathrm{mg} / \mathrm{dL}$.

The patient returned to the clinic for the second Viscum album intravenous application, and the mass had surprisingly reduced about $90 \%$ of its total size, compared to the initial lesion, remaining only a slight edema on the upper lip (Figure 3). The tutor reported

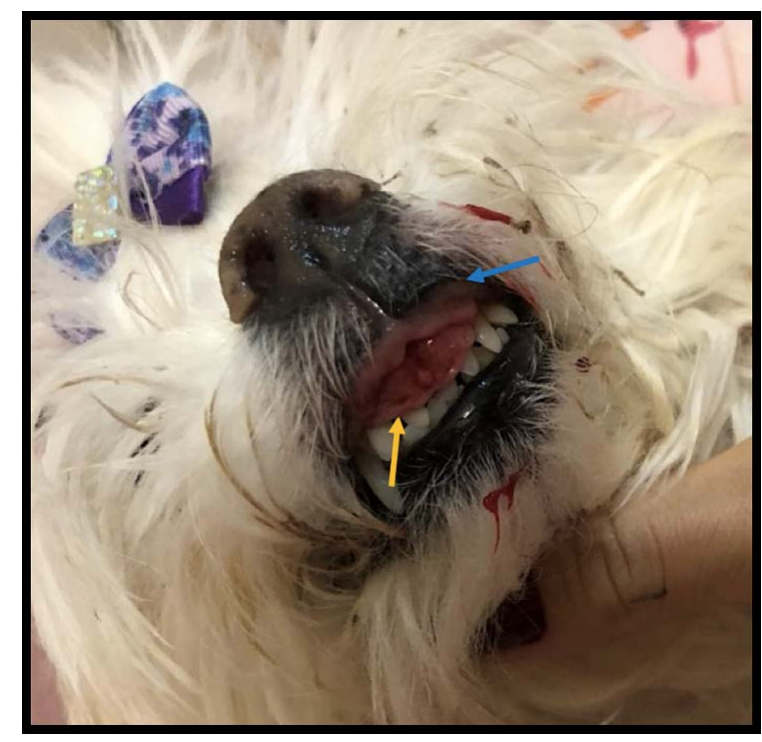

Figure 2: Appearance of the initial lesion. Blue arrow: increased volume of the upper lip with edema. Yellow arrow: mass adhered between upper lip and gum, diagnosed by biopsy and histopathology as squamous cell carcinoma.

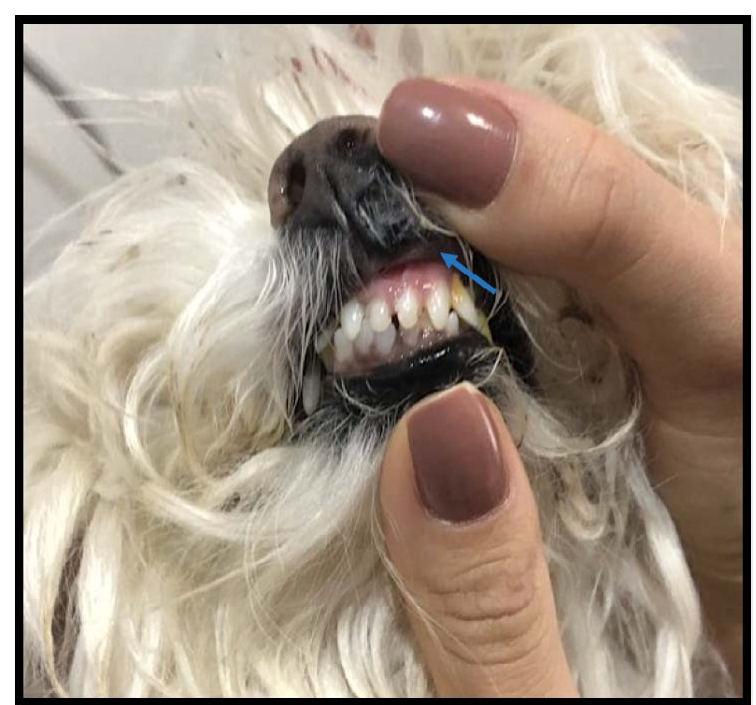

Figure 3: Appearance of the lesion after seven days of treatment with Viscum album subcutaneous applications on alternate days. Blue arrow: residual edema in the upper lip. 


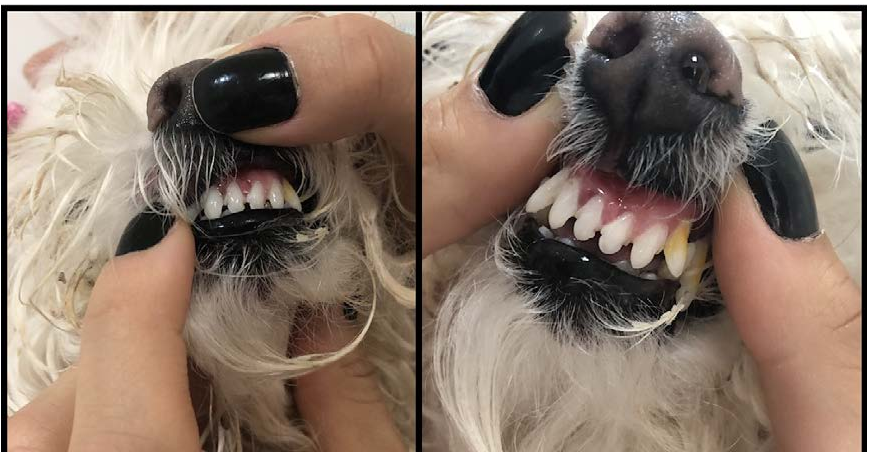

Figure 4: Appearance of the region previously diagnosed with SCC. Result of the treatment 30 days after Viscum album applications. Both images demonstrate the disappearance of the tissue between the upper lip and gum, previously seen, and the edema reduction in the superior lip.

improvement in the animal's overall condition, improved appetite, sleep quality, and mood. The animal also returned to show interest in playing as she previously used to do. After 30 days of treatment, the affected site was completely restored (Figure 4).

Eight months after the complete reestablishment, the animal returned to the clinic for dental prophylaxis. On this occasion, a new fragment was collected of the region previously affected and diagnosed as SCC. The sample was sent for histopathology analysis to the VetPat Laboratory in Campinas, SP, Brazil. The result showed the presence of fibroconjunctive tissue with the prevalence of scar tissue. Thus, the medication (Viscum album D3) was maintained, but only once a day, three times a week, subcutaneously. However, the animal died (acute renal disease) six months after the dental prophylaxis procedure, at 17 years.

\section{Discussion}

Cancer is the primary cause of death or euthanasia in veterinary patients. The oral cavity is in fourth place with the highest incidence, being behind only the mammary gland, genitals, and skin. In general, oral tumors are only noticed when the disease is already in an advanced clinical stage $[9,10]$.

Among oral neoplasms, SCC has been considered a malignant and ulcerative epidermal tumor with a poor prognosis, depending on the stage of the disease evolution [11]. SCC is considered the second most common oral malignant neoplasm in dogs [2] and one of the most commonly diagnosed tumors in humans [12]. It has already been described in the lips, gums, tonsils, oral mucosa, and tongue [13,14]. In many cases, metastasis occurs by lymphatic vessels, mainly to the mandibular region and/or retropharyngeal lymph nodes and lungs [14]. However, in the case here reported, no sign of metastasis was observed in the physical evaluations performed while the animal was monitored until the day of its death. This fact could probably be due to the well-differentiated characteristic of the tumor since metastasis seems to be related to the differentiation degree of neoplastic cells, the most likely occurrence in tumors with little differentiation [15].

The treatment for cancer patients aims primarily at the eradication of the affected tissue, prioritizing the preservation of the functional structure of the affected area, and when possible, its aesthetic appearance [10]. Several options are available for treating this disease in small animals, such as surgical excision, systemic and/or intralesional chemotherapy, radiotherapy, cryosurgery, and immunotherapy [16]. According to Wiggs and Lobprise [17] and Gioso [18], surgery therapy is the most efficient among these options.

However, most conventional treatments do not contemplate the total cure of the disease, nor do they excel in the patient's life quality, causing, several times, various side effects and the occurrence of metastases. In contrast to the therapies of choice or conventional therapies, this report affirms the effectiveness of treatment here used for treating SCC, which did not have side effects on the patient and resulted in the total cure of the disease, improving the patient's quality of life, as stated by the tutor.

According to Gioso [18], a favorable prognosis with no or reduced tumor recurrences would demand surgical resection with a wide safety margin. Such procedures may cause significant deformations, mutilations, altering the functional structure of the organ affected. There are also complications associated with the postoperative period of maxillary or mandibular resection, which may include anorexia, tongue projection, difficulty in grasping food, palatal ulcer due to malocclusion, dehiscence of the surgical wound, infection, oronasal fistula, epistaxis, disorders in the salivary gland ducts, and excessive salivation [19]. Contrasting these authors, the prognosis of the present case indicated that depending on the tumor stage the Viscum album therapy should be considered a real option for treating cancer patients, either as a palliative treatment or even aiming at the patient's cure.

The findings here reported corroborate with Valle and Carvalho [6], who referred to the healing of ulcerated lesions caused by SCC in the breast in a patient in palliative care. However, this study not only showed the resolution of an ulcerated lesion but also demonstrated the complete cure of the process, which was confirmed by an incisional biopsy performed ten months after the initial SCC diagnosis and by the absence of metastasis recurrence.

\section{Conclusion}

These data meet the need for new treatment options for cancer patients at different disease stages. The ultra-diluted Viscum album has been shown to be an excellent treatment option for these patients, whether in search of a cure for cancer or just in search of palliative care. Further studies are still needed to confirm the effectiveness of such effects in patients in the various stages of the disease.

\section{Conflict of Interest}

The authors declare that there is no conflict of interest in this case report.

\section{References}

1. Webb JL, Burns RE, Brown HM, LeRoy BE, Kosarek CE (2009) Squamous cell carcinoma. Compend Contin Educ Vet 31: 133-142.

2. Gardner DG (1996) Spontaneous squamous cell carcinomas of the oral region in domestic animals: a review and consideration of their relevance to human research. Oral Dis 2: 148-154. [crossref]

3. Waldman A, Schmults C (2019) Cutaneous Squamous Cell Carcinoma. Hematol Oncol Clin North Am 33: 1. 
4. Thomson PJ (2018) Perspectives on oral squamous cell carcinoma preventionproliferation, position, progression and prediction. J Oral Pathol Med 47: 803-807. [crossref]

5. Bilgic O, Duda L, Sánchez MD, Lewis JR (2015) Feline Oral Squamous Cell Carcinoma: Clinical Manifestations and Literature Review. J Vet Dent 32: 30-40. [crossref]

6. Valle ACV, Carvalho AC (2021) Homeopathic Viscum Album on the Treatment of Scamous Cell Carcinoma Lesion in a Dog (Canis familiaris) - Case Report. Integr J Vet Biosc 5: 1-3.

7. Valle ACV, Carvalho AC, Andrade RV (2021) Viscum Album - Literature Review. Int J Sci Res 10: 63-71.

8. Valle ACV (2020) In vitro and in vivo evaluation of the ultra-diluted Viscum album efficacy and safety. Doctorate dissertation. Catholic University of Brasilia - UCB, Brasilia-DF, Brazil, 78.

9. Cotran RS, Kumar V, Collins T (2000) Neoplasia. In: Structural and functional pathology. 6th ed. Rio de Janeiro: Guanabara Koogan 233-241.

10. Howard PE (2002) Maxillary and mandibular neoplasms. In Bichard SJ, Sherding RG (Eds) Saunders Manual - Small animal clinic. 2nd ed. Roca: São Paulo 1181-1189.

11. Rodaski S, Werner J (2008) Skin neoplasms. In Daleck CR, De Nardi AB, Rodaski S (Eds) Oncology in dogs and cats. Roca: São Paulo 253-279.
12. Uma RS, Naresh KN, D’Cruz AK, Mulherkar R, Borges AM (2007) Metastasis of squamous cell carcinoma of the oral tongue is associated with down-regulation of epidermal fatty acid binding protein (E-FABP). Oral Oncol 43: 27-32. [crossref]

13. Dhaliwal RS, Kitchell BE, Maretta SM (1998) Oral tumors in dogs and cats. Part I. Diagnosis and clinical signs. Compend Continuing Educ Vet 20: 1011-1021.

14. Meuten DJ (2002) Tumors of the alimentary tract. Tumors of domestic animals. 4th ed. Iowa: Iowa State Press 509-546.

15. Denis MM, Ehrhart N, Duncan CG, Barnes AB, Ehrhart EJ (2006) Frequency and risk factors associated with lingual lesions in dogs: 1,196 cases (1995-2004). J Am Vet Med Assoc 228: 1533-1537. [crossref]

16. Mayer MN, Anthony JM (2007) Radiation therapy for oral tumors: Canine acanthomatous ameloblastoma. Can Vet J 48: 99-101. [crossref]

17. Wiggs RB, Lobprise HB (1997) Veterinary Dentistry, Principles and Practice. Philadelphia: Lippncott-Raven 748.

18. Gioso MA (2007) Neoplasia da cavidade oral. In: Veterinary odontology for the small animal clinician. 2nd ed. Sao Paulo: Manole 91-100.

19. Kessler M (2006) Mandibulectomy and maxillectomy as a treatment for bone invasive oral neoplasia in the dog - a retrospective analysis of 31 patients. Europ J Comp Anim Pract 16: 73-82.

\section{Citation:}

Viana Valle AC, de Carvalho AC (2021) Squamous Cell Carcinoma in a Dog (Canis familiaris) Treated by the Viscum album Therapy - Case Report. Integr J Vet Biosci Volume 5(3): 1-4 